\title{
On certain combinatorial expansions of the Legendre-Stirling numbers
}

\author{
Shi-Mei Ma* \\ School of Mathematics and Statistics \\ Northeastern University at Qinhuangdao \\ Hebei, P.R. China \\ shimeimapapers@163.com \\ Jun $\mathrm{Ma}^{\dagger}$ \\ Department of Mathematics \\ Shanghai Jiao Tong University \\ Shanghai, P.R. China \\ majun904@sjtu.edu.cn \\ Yeong-Nan Yeh ${ }^{\ddagger}$ \\ Institute of Mathematics \\ Academia Sinica \\ Taipei, Taiwan \\ mayeh@math.sinica.edu.tw
}

Submitted: Aug 5, 2018; Accepted: Dec 2,2018; Published: Dec 21, 2018

(C) The authors. Released under the CC BY-ND license (International 4.0).

\begin{abstract}
The Legendre-Stirling numbers of the second kind were introduced by Everitt et al. in the spectral theory of powers of the Legendre differential expressions. As a continuation of the work of Andrews and Littlejohn (Proc. Amer. Math. Soc., 137 (2009), 2581-2590), we provide a combinatorial code for Legendre-Stirling set partitions. As an application, we obtain expansions of the Legendre-Stirling numbers of both kinds in terms of binomial coefficients.
\end{abstract}

Mathematics Subject Classifications: 05A18, 05A19

\section{Introduction}

The study on Legendre-Stirling numbers and Jacobi-Stirling numbers has become an active area of research in the past decade. In particular, these numbers are closely related to set partitions [3], symmetric functions [12], special functions [11] and so on.

Let $\ell[y](t)=-\left(1-t^{2}\right) y^{\prime \prime}(t)+2 t y^{\prime}(t)$ be the Legendre differential operator. Then the Legendre polynomial $y(t)=P_{n}(t)$ is an eigenvector for the differential operator $\ell$

*Supported by Natural Science Foundation of Hebei Province (A2017501007) and NSFC (11401083).

${ }^{\dagger}$ Supported by NSFC 11571235 .

${ }^{\ddagger}$ Supported by NSC 107-2115-M-001-009-MY3. 
corresponding to $n(n+1)$, i.e., $\ell[y](t)=n(n+1) y(t)$. Following Everitt et al. [6], for $n \in \mathbb{N}$, the Legendre-Stirling numbers LS $(n, k)$ of the second kind appeared originally as the coefficients in the expansion of the $n$-th composite power of $\ell$, i.e.,

$$
\ell^{n}[y](t)=\sum_{k=0}^{n}(-1)^{k} \operatorname{LS}(n, k)\left(\left(1-t^{2}\right)^{k} y^{(k)}(t)\right)^{(k)} .
$$

For each $k \in \mathbb{N}$, Everitt et al. [6, Theorem 4.1)] obtained that

$$
\prod_{r=0}^{k} \frac{1}{1-r(r+1) x}=\sum_{n=0}^{\infty} \operatorname{LS}(n, k) x^{n-k}, \quad\left(|x|<\frac{1}{k(k+1)}\right) .
$$

According to [2, Theorem 5.4], the numbers LS $(n, k)$ have the following horizontal generating function

$$
x^{n}=\sum_{k=0}^{n} \operatorname{LS}(n, k) \prod_{i=0}^{k-1}(x-i(1+i)) .
$$

It follows from $(2)$ that the numbers LS $(n, k)$ satisfy the recurrence relation

$$
\operatorname{LS}(n, k)=\operatorname{LS}(n-1, k-1)+k(k+1) \operatorname{LS}(n-1, k) .
$$

with the initial conditions $\operatorname{LS}(n, 0)=\delta_{n, 0}$ and $\operatorname{LS}(0, k)=\delta_{0, k}$, where $\delta_{i, j}$ is the Kronecker's symbol. By using (1), Andrews et al. [2, Theorem 5.2] derived that the numbers LS $(n, k)$ satisfy the vertical recurrence relation

$$
\operatorname{LS}(n, j)=\sum_{k=j}^{n} \operatorname{LS}(k-1, j-1)(j(j+1))^{n-k} .
$$

Following [7, Theorem 4.1], the Jacobi-Stirling number $\operatorname{JS}_{n}^{k}(z)$ of the second kind may be defined by

$$
x^{n}=\sum_{k=0}^{n} \operatorname{JS}_{n}^{k}(z) \prod_{i=0}^{k-1}(x-i(z+i)) .
$$

It follows from (3) that the numbers $\operatorname{JS}_{n}^{k}(z)$ satisfy the recurrence relation

$$
\operatorname{JS}_{n}^{k}(z)=\operatorname{JS}_{n-1}^{k-1}(z)+k(k+z) \operatorname{JS}_{n-1}^{k}(z),
$$

with the initial conditions $\operatorname{JS}_{n}^{0}(z)=\delta_{n, 0}$ and $\operatorname{JS}_{0}^{k}(z)=\delta_{0, k}$ (see [11] for instance). It is clear that $\operatorname{JS}_{n}^{k}(1)=\mathrm{LS}(n, k)$. In [9], Gessel, Lin and Zeng studied generating function of the coefficients of $\operatorname{JS}_{n+k}^{n}(z)$. Note that $\operatorname{JS}_{n+k}^{n}(1)=\operatorname{LS}(n+k, n)$. This paper is devoted to the following problem.

Problem 1. Let $k$ be a given nonnegative integer. Could the numbers LS $(n+k, n)$ be expanded in the binomial basis? 
A particular value of $\operatorname{LS}(n, k)$ is provided at the end of [3]:

$$
\operatorname{LS}(n+1, n)=2\left(\begin{array}{c}
n+2 \\
3
\end{array}\right) \text {. }
$$

In [5, Eq. (19)], Egge obtained that

$$
\mathrm{LS}(n+2, n)=40\left(\begin{array}{c}
n+2 \\
6
\end{array}\right)+72\left(\begin{array}{c}
n+2 \\
5
\end{array}\right)+36\left(\begin{array}{c}
n+2 \\
4
\end{array}\right)+4\left(\begin{array}{c}
n+2 \\
3
\end{array}\right) .
$$

Using the triangular recurrence relation $\left(\begin{array}{c}n+1 \\ k\end{array}\right)=\left(\begin{array}{c}n \\ k\end{array}\right)+\left(\begin{array}{c}n \\ k-1\end{array}\right)$, we get

$$
\operatorname{LS}(n+2, n)=40\left(\begin{array}{c}
n+3 \\
6
\end{array}\right)+32\left(\begin{array}{c}
n+3 \\
5
\end{array}\right)+4\left(\begin{array}{c}
n+3 \\
4
\end{array}\right) .
$$

Egge [5, Theorem 3.1] showed that for $k \geqslant 0$, the quantity LS $(n+k, n)$ is a polynomial of degree $3 k$ in $n$ with leading coefficient $\frac{1}{3^{k} k !}$.

As a continuation of [3] and [5], in Section 2, we give a solution of Problem 1. Moreover, we get an expansion of the Legendre-Stirling numbers of the first kind in terms of binomial coefficients.

\section{Main results}

The combinatorial interpretation of the Legendre-Stirling numbers LS $(n, k)$ of the second kind was first given by Andrews and Littlejohn [3]. For $n \geqslant 1$, let $\mathrm{M}_{n}$ denote the multiset $\{1, \overline{1}, 2, \overline{2}, \ldots, n, \bar{n}\}$, in which we have one unbarred copy and one barred copy of each integer $i$, where $1 \leqslant i \leqslant n$. Throughout this paper, we always assume that the elements of $\mathrm{M}_{n}$ are ordered by

$$
\overline{1}=1<\overline{2}=2<\cdots<\bar{n}=n .
$$

A Legendre-Stirling set partition of $\mathrm{M}_{n}$ is a set partition of $\mathrm{M}_{n}$ with $k+1$ blocks $B_{0}, B_{1}, \ldots, B_{k}$ and with the following rules:

$\left(r_{1}\right)$ The 'zero box' $B_{0}$ is the only box that may be empty and it may not contain both copies of any number;

$\left(r_{2}\right)$ The 'nonzero boxes' $B_{1}, B_{2}, \ldots, B_{k}$ are indistinguishable and each is non-empty. For any $i \in[k]$, the box $B_{i}$ contains both copies of its smallest element and does not contain both copies of any other number.

Let $\mathcal{L} \mathcal{S}(n, k)$ denote the set of Legendre-Stirling set partitions of $\mathrm{M}_{n}$ with one zero box and $k$ nonzero boxes. The standard form of an element of $\mathcal{L} \mathcal{S}(n, k)$ is written as

$$
\sigma=B_{1} B_{2} \cdots B_{k} B_{0}
$$


where $B_{0}$ is the zero box and the minima of $B_{i}$ is less than that of $B_{j}$ when $1 \leqslant i<j \leqslant k$. Clearly, the minima of $B_{1}$ are 1 and $\overline{1}$. Throughout this paper we always write $\sigma \in$ $\mathcal{L S}(n, k)$ in the standard form. As usual, we let angle bracket symbol $\langle i, j, \ldots>$ and curly bracket symbol $\{k, \bar{k}, \ldots\}$ denote the zero box and nonzero box, respectively. In particular, let $\langle>$ denote the empty zero box. For example, $\{1, \overline{1}, 3\}\{2, \overline{2}\}<\overline{3}>\in \mathcal{L} \mathcal{S}(3,2)$. A classical result of Andrews and Littlejohn [3, Theorem 2] says that

$$
\operatorname{LS}(n, k)=\# \mathcal{L S}(n, k) \text {. }
$$

We now provide a combinatorial code for Legendre-Stirling partitions (CLS -sequence for short).

Definition 2. We call $Y_{n}=\left(y_{1}, y_{2}, \ldots, y_{n}\right)$ a CLS -sequence of length $n$ if $y_{1}=X$ and

$$
y_{k+1} \in\left\{X, A_{i, j}, B_{s}, \bar{B}_{s}, 1 \leqslant i, j, s \leqslant n_{x}\left(Y_{k}\right), i \neq j\right\} \quad \text { for } k=1,2, \ldots, n-1,
$$

where $n_{x}\left(Y_{k}\right)$ is the number of the symbol $X$ in $Y_{k}=\left(y_{1}, y_{2}, \ldots, y_{k}\right)$.

For example, $\left(X, X, A_{1,2}\right)$ is a CLS -sequence, while $\left(X, X, A_{1,2}, B_{3}\right)$ is not since $y_{4}=B_{3}$ and $3>n_{x}\left(Y_{3}\right)=2$. Let $\mathcal{C} \mathcal{L} \mathcal{S}_{n}$ denote the set of CLS -sequences of length $n$.

The following lemma is a fundamental result.

Lemma 3. For $n \geqslant 1$, we have $\operatorname{LS}(n, k)=\#\left\{Y_{n} \in \mathcal{C} \mathcal{L} \mathcal{S}_{n} \mid n_{x}\left(Y_{n}\right)=k\right\}$.

Proof. Let $\mathcal{C} \mathcal{L S}(n, k)=\left\{Y_{n} \in \mathcal{C} \mathcal{L} \mathcal{S}_{n} \mid n_{x}\left(Y_{n}\right)=k\right\}$. Now we start to construct a bijection, denoted by $\Phi$, between $\mathcal{L S}(n, k)$ and $\mathcal{C} \mathcal{L} \mathcal{S}(n, k)$. When $n=1$, we have $y_{1}=X$. Set $\Phi\left(Y_{1}\right)=\{1, \overline{1}\}<>$. This gives a bijection from $\mathcal{C} \mathcal{L} \mathcal{S}(1,1)$ to $\mathcal{L} \mathcal{S}(1,1)$. Let $n=m$. Suppose $\Phi$ is a bijection from $\mathcal{C} \mathcal{L S}(n, k)$ to $\mathcal{C} \mathcal{L} \mathcal{S}(n, k)$ for all $k$. Consider the case $n=$ $m+1$. Let

$$
Y_{m+1}=\left(y_{1}, y_{2}, \ldots, y_{m}, y_{m+1}\right) \in \mathcal{C} \mathcal{L S}_{m+1} .
$$

Then $Y_{m}=\left(y_{1}, y_{2}, \ldots, y_{m}\right) \in \mathcal{C} \mathcal{L S}(m, k)$ for some $k$. Assume $\Phi\left(Y_{m}\right) \in \mathcal{L} \mathcal{S}(m, k)$. Consider the following three cases:

(i) If $y_{m+1}=X$, then let $\Phi\left(Y_{m+1}\right)$ be obtained from $\Phi\left(Y_{m}\right)$ by putting the box $\{m+$ $1, \overline{m+1}\}$ just before the zero box. In this case, $\Phi\left(Y_{m+1}\right) \in \mathcal{L} \mathcal{S}(m+1, k+1)$.

(ii) If $y_{m+1}=A_{i, j}$, then let $\Phi\left(Y_{m+1}\right)$ be obtained from $\Phi\left(Y_{m}\right)$ by inserting the entry $m+1$ to the $i$ th nonzero box and inserting the entry $\overline{m+1}$ to the $j$ th nonzero box. In this case, $\Phi\left(Y_{m+1}\right) \in \mathcal{L} \mathcal{S}(m+1, k)$.

(iii) If $y_{m+1}=B_{s}$ (resp. $\left.y_{m+1}=\bar{B}_{s}\right)$, then let $\Phi\left(Y_{m+1}\right)$ be obtained from $\Phi\left(Y_{m}\right)$ by inserting the entry $m+1$ (resp. $\overline{m+1}$ ) to the $s$ th nonzero box and inserting the entry $\overline{m+1}$ (resp. $m+1$ ) to the zero box. In this case, $\Phi\left(Y_{m+1}\right) \in \mathcal{L} \mathcal{S}(m+1, k)$.

After the above step, it is clear that the obtained $\Phi\left(Y_{m+1}\right)$ is in standard form. By induction, we see that $\Phi$ is the desired bijection from $\mathcal{C} \mathcal{L} \mathcal{S}(n, k)$ to $\mathcal{C} \mathcal{L} \mathcal{S}(n, k)$, which also gives a constructive proof of Lemma 3 . 
Example 4. Let $Y_{5}=\left(X, X, A_{2,1}, B_{2}, \bar{B}_{1}\right)$. The correspondence between $Y_{5}$ and $\Phi\left(Y_{5}\right)$ is built up as follows:

$$
\begin{aligned}
X & \Leftrightarrow\{1, \overline{1}\}<>; \\
X & \Leftrightarrow\{1, \overline{1}\}\{2, \overline{2}\}<>; \\
A_{2,1} & \Leftrightarrow\{1, \overline{1}, \overline{3}\}\{2, \overline{2}, 3\}<>; \\
B_{2} & \Leftrightarrow\{1, \overline{1}, \overline{3}\}\{2, \overline{2}, 3,4\}<\overline{4}>; \\
\bar{B}_{1} & \Leftrightarrow\{1, \overline{1}, \overline{3}, \overline{5}\}\{2, \overline{2}, 3,4\}<\overline{4}, 5>.
\end{aligned}
$$

As an application of Lemma 3, we present the following lemma.

Lemma 5. Let $k$ be a given positive integer. Then for $n \geqslant 1$, we have

$$
\operatorname{LS}(n+k, n)=2^{k} \sum_{t_{k}=1}^{n}\left(\begin{array}{c}
t_{k}+1 \\
n
\end{array}\right) \sum_{t_{k-1}=1}^{t_{k}}\left(\begin{array}{c}
t_{k-1}+1 \\
2
\end{array}\right) \cdots \sum_{t_{2}=1}^{t_{3}}\left(\begin{array}{c}
t_{2}+1 \\
2
\end{array}\right) \sum_{t_{1}=1}^{t_{2}}\left(\begin{array}{c}
t_{1}+1 \\
2
\end{array}\right) .
$$

Proof. It follows from Lemma 3 that

$$
\operatorname{LS}(n+k, n)=\#\left\{Y_{n+k} \in \mathcal{C} \mathcal{L} \mathcal{S}_{n+k} \mid n_{x}\left(Y_{n+k}\right)=n\right\} .
$$

Let $Y_{n+k}=\left(y_{1}, y_{2}, \ldots, y_{n+k}\right)$ be a given element in $\mathcal{C} \mathcal{L} \mathcal{S}_{n+k}$. Since $n_{x}\left(Y_{n+k}\right)=n$, it is natural to assume that $y_{i}=X$ except $i=t_{1}+1, t_{2}+2, \cdots, t_{k}+k$. Let $\sigma$ be the corresponding Legendre-Stirling partition of $Y_{n+k}$. For $1 \leqslant \ell \leqslant k$, consider the value of $y_{t_{\ell}+\ell}$. Note that the number of the symbols $X$ before $y_{t_{\ell}+\ell}$ is $t_{\ell}$. Let $\widehat{\sigma}$ be the corresponding Legendre-Stirling set partition of $\left(y_{1}, y_{2}, \ldots, y_{t_{\ell}+\ell-1}\right)$. Now we insert $y_{t_{\ell}+\ell}$. We distinguish two cases:

(i) If $y_{t_{\ell}+\ell}=A_{i, j}$, then we should insert the entry $t_{\ell}+\ell$ to the $i$ th nonzero box of $\widehat{\sigma}$ and insert $\overline{t_{\ell}+\ell}$ to the $j$ th nonzero box. This gives $2\left(\begin{array}{c}t_{\ell} \\ 2\end{array}\right)$ possibilities, since $1 \leqslant i, j \leqslant t_{\ell}$ and $i \neq j$.

(ii) If $y_{t_{\ell}+\ell}=B_{s}$ (resp. $y_{t_{\ell}+\ell}=\bar{B}_{s}$ ), then we should insert the entry $t_{\ell}+\ell$ (resp. $\overline{t_{\ell}+\ell}$ ) to the $s$ th nonzero box of $\widehat{\sigma}$ and insert $\overline{t_{\ell}+\ell}$ (resp. $t_{\ell}+\ell$ ) to the zero box. This gives $2\left(\begin{array}{c}t_{\ell} \\ 1\end{array}\right)$ possibilities, since $1 \leqslant s \leqslant t_{\ell}$.

Therefore, there are exactly $2\left(\begin{array}{c}t_{\ell} \\ 2\end{array}\right)+2\left(\begin{array}{c}t_{\ell} \\ 1\end{array}\right)=2\left(\begin{array}{c}t_{\ell}+1 \\ 2\end{array}\right)$ Legendre-Stirling set partitions of $\mathrm{M}_{t_{\ell}+\ell}$ can be generated from $\widehat{\sigma}$ by inserting the entry $y_{t_{\ell}+\ell}$. Note that $1 \leqslant t_{j-1} \leqslant t_{j} \leqslant n$ for $2 \leqslant j \leqslant k$. Applying the product rule for counting, we immediately get $(6)$.

The following simple result will be used in our discussion.

Lemma 6. Let $a$ and $b$ be two given integers. Then

$$
\left(\begin{array}{c}
x-b \\
2
\end{array}\right)\left(\begin{array}{l}
x \\
a
\end{array}\right)=\left(\begin{array}{c}
a+2 \\
2
\end{array}\right)\left(\begin{array}{c}
x \\
a+2
\end{array}\right)+(a+1)(a-b)\left(\begin{array}{c}
x \\
a+1
\end{array}\right)+\left(\begin{array}{c}
a-b \\
2
\end{array}\right)\left(\begin{array}{l}
x \\
a
\end{array}\right) .
$$

In particular,

$$
\left(\begin{array}{c}
x-1 \\
2
\end{array}\right)\left(\begin{array}{l}
x \\
a
\end{array}\right)=\left(\begin{array}{c}
a+2 \\
2
\end{array}\right)\left(\begin{array}{c}
x \\
a+2
\end{array}\right)+\left(a^{2}-1\right)\left(\begin{array}{c}
x \\
a+1
\end{array}\right)+\left(\begin{array}{c}
a-1 \\
2
\end{array}\right)\left(\begin{array}{l}
x \\
a
\end{array}\right) .
$$


Proof. Note that

$$
\left(\begin{array}{c}
a+2 \\
2
\end{array}\right) \frac{(x-a)(x-a-1)}{(a+2)(a+1)}+(a+1)(a-b) \frac{x-a}{a+1}+\left(\begin{array}{c}
a-b \\
2
\end{array}\right)=\left(\begin{array}{c}
x-b \\
2
\end{array}\right) .
$$

This yields the desired result.

We can now conclude the main result of this paper from the discussion above.

Theorem 7. Let $k$ be a given nonnegative integer. For $n \geqslant 1$, the numbers $\operatorname{LS}(n+k, n)$ can be expanded in the binomial basis as

$$
\mathrm{LS}(n+k, n)=2^{k} \sum_{i=k+2}^{3 k} \gamma(k, i)\left(\begin{array}{c}
n+k+1 \\
i
\end{array}\right),
$$

where the coefficients $\gamma(k, i)$ are all positive integers for $k+2 \leqslant i \leqslant 3 k$ and satisfy the recurrence relation

$$
\gamma(k+1, i)=\left(\begin{array}{c}
i-k-1 \\
2
\end{array}\right) \gamma(k, i-1)+(i-1)(i-k-2) \gamma(k, i-2)+\left(\begin{array}{c}
i-1 \\
2
\end{array}\right) \gamma(k, i-3),
$$

with the initial conditions $\gamma(0,0)=1, \gamma(0, i)=\gamma(i, 0)=0$ for $i \neq 0$. Let $\gamma_{k}(x)=$ $\sum_{i=k+2}^{3 k} \gamma(k, i) x^{i}$. Then the polynomials $\gamma_{k}(x)$ satisfy the recurrence relation

$\gamma_{k+1}(x)=\left(\frac{k(k+1)}{2}-k x+x^{2}\right) x \gamma_{k}(x)-\left(k+(k-2) x-2 x^{2}\right) x^{2} \gamma_{k}^{\prime}(x)+\frac{(1+x)^{2} x^{3}}{2} \gamma_{k}^{\prime \prime}(x)$,

with the initial conditions $\gamma_{0}(x)=1, \gamma_{1}(x)=x^{3}$ and $\gamma_{2}(x)=x^{4}+8 x^{5}+10 x^{6}$.

Proof. We prove (7) by induction on $k$. It is clear that $\operatorname{LS}(n, n)=1=\left(\begin{array}{c}n+1 \\ 0\end{array}\right)$. When $k=1$, by using the Chu Shih-Chieh's identity

$$
\left(\begin{array}{l}
n+1 \\
k+1
\end{array}\right)=\sum_{i=k}^{n}\left(\begin{array}{l}
i \\
k
\end{array}\right)
$$

we obtain

$$
\sum_{t_{1}=1}^{n}\left(\begin{array}{c}
t_{1}+1 \\
2
\end{array}\right)=\left(\begin{array}{c}
n+2 \\
3
\end{array}\right),
$$

and so (4) is established. When $k=2$, it follows from Lemma 5 that

$$
\begin{aligned}
\operatorname{LS}(n+2, n) & =4 \sum_{t_{2}=1}^{n}\left(\begin{array}{c}
t_{2}+1 \\
2
\end{array}\right) \sum_{t_{1}=1}^{t_{2}}\left(\begin{array}{c}
t_{1}+1 \\
2
\end{array}\right) \\
& =4 \sum_{t_{2}=1}^{n}\left(\begin{array}{c}
t_{2}+1 \\
2
\end{array}\right)\left(\begin{array}{c}
t_{2}+2 \\
3
\end{array}\right) .
\end{aligned}
$$


Setting $x=t_{2}+2$ and $a=3$ in Lemma 6 , we get

$$
\begin{aligned}
\operatorname{LS}(n+2, n) & =4 \sum_{t_{2}=1}^{n}\left(10\left(\begin{array}{c}
t_{2}+2 \\
5
\end{array}\right)+8\left(\begin{array}{c}
t_{2}+2 \\
4
\end{array}\right)+\left(\begin{array}{c}
t_{2}+2 \\
3
\end{array}\right)\right) \\
& =4\left(10\left(\begin{array}{c}
n+3 \\
6
\end{array}\right)+8\left(\begin{array}{c}
n+3 \\
5
\end{array}\right)+\left(\begin{array}{c}
n+3 \\
4
\end{array}\right)\right),
\end{aligned}
$$

which yields (5). Along the same lines, it is not hard to verify that

$$
\begin{aligned}
\operatorname{LS}(n+3, n) & =8 \sum_{t_{3}=1}^{n}\left(\begin{array}{c}
t_{3}+1 \\
2
\end{array}\right)\left(10\left(\begin{array}{c}
t_{3}+3 \\
6
\end{array}\right)+8\left(\begin{array}{c}
t_{3}+3 \\
5
\end{array}\right)+\left(\begin{array}{c}
t_{3}+3 \\
4
\end{array}\right)\right) \\
& =8\left(280\left(\begin{array}{c}
n+4 \\
9
\end{array}\right)+448\left(\begin{array}{c}
n+4 \\
8
\end{array}\right)+219\left(\begin{array}{c}
n+4 \\
7
\end{array}\right)+34\left(\begin{array}{c}
n+4 \\
6
\end{array}\right)+\left(\begin{array}{c}
n+4 \\
5
\end{array}\right)\right) .
\end{aligned}
$$

Hence the formula ( 7 ) holds for $k=0,1,2,3$, so we proceed to the inductive step. For $k \geqslant 3$, assume that

$$
\operatorname{LS}(n+k, n)=2^{k} \sum_{i=k+2}^{3 k} \gamma(k, i)\left(\begin{array}{c}
n+k+1 \\
i
\end{array}\right) .
$$

It follows from Lemma 5 that

$$
\operatorname{LS}(n+k+1, n)=2^{k+1} \sum_{t_{k+1}=1}^{n}\left(\begin{array}{c}
t_{k+1}+1 \\
2
\end{array}\right) \sum_{i=k+2}^{3 k} \gamma(k, i)\left(\begin{array}{c}
t_{k+1}+k+1 \\
i
\end{array}\right)
$$

By using Lemma 6 and the Chu Shih-Chieh's identity, it is routine to verify that the coefficients $\gamma(k, i)$ satisfy the recurrence relation (8), and so (7) is established for general $k$. Multiplying both sides of (8) by $x^{i}$ and summing for all $i$, we immediately get (9).

In [2], Andrews et al. introduced the (unsigned) Legendre-Stirling numbers Lc $(n, k)$ of the first kind, which may be defined by the recurrence relation

$$
\operatorname{Lc}(n, k)=\operatorname{Lc}(n-1, k-1)+n(n-1) \operatorname{Lc}(n-1, k),
$$

with the initial conditions $\operatorname{Lc}(n, 0)=\delta_{n, 0}$ and $\operatorname{Lc}(0, n)=\delta_{0, n}$. Let $f_{k}(n)=\operatorname{LS}(n+k, n)$. According to Egge [5, Eq. (23)], we have

$$
\operatorname{Lc}(n-1, n-k-1)=(-1)^{k} f_{k}(-n)
$$

for $k \geqslant 0$. For $m, k \in \mathbb{N}$, we define

$$
\left(\begin{array}{c}
-m \\
k
\end{array}\right)=\frac{(-m)(-m-1) \cdots(-m-k+1)}{k !} .
$$

Combining (7) and (10), we immediately get the following result. 
Corollary 8. Let $k$ be a given nonnegative integer. For $n \geqslant 1$, the numbers $\operatorname{Lc}(n-1, n-$ $k-1)$ can be expanded in the binomial basis as

$$
\operatorname{Lc}(n-1, n-k-1)=(-1)^{k} 2^{k} \sum_{i=k+2}^{3 k} \gamma(k, i)\left(\begin{array}{c}
-n+k+1 \\
i
\end{array}\right),
$$

where the coefficients $\gamma(k, i)$ are defined by $(8)$.

It follows from (9) that

$$
\begin{aligned}
\gamma(k+1, k+3) & =\left(\frac{k(k+1)}{2}-k(k+2)+\frac{(k+2)(k+1)}{2}\right) \gamma(k, k+2), \\
\gamma(k+1,3 k+3) & =\left(1+6 k+\frac{3 k(3 k-1)}{2}\right) \gamma(k, 3 k), \\
\gamma_{k+1}(-1) & =-\left(\frac{k(k+1)}{2}+k+1\right) \gamma_{k}(-1) .
\end{aligned}
$$

Since $\gamma(1,3)=1$ and $\gamma_{1}(-1)=-1$, it is easy to verify that for $k \geqslant 1$, we have

$$
\gamma(k, k+2)=1, \gamma(k, 3 k)=\frac{(3 k) !}{k !(3 !)^{k}}, \gamma_{k}(-1)=(-1)^{k} \frac{(k+1) ! k !}{2^{k}} .
$$

It should be noted that the number $\gamma(k, 3 k)$ is the number of partitions of $\{1,2, \ldots, 3 k\}$ into blocks of size 3 , and the number $\frac{(k+1) ! k !}{2^{k}}$ is the product of first $k$ positive triangular numbers. Moreover, if the number $\operatorname{LS}(n+k, n)$ is viewed as a polynomial in $n$, then its degree is $3 k$, which is implied by the quantity $\left(\begin{array}{c}n+k+1 \\ 3 k\end{array}\right)$. Furthermore, the leading coefficient of $\operatorname{LS}(n+k, n)$ is given by $2^{k} \gamma(k, 3 k) \frac{1}{(3 k) !}=2^{k} \frac{(3 k) !}{k !(3 !)^{k}} \frac{1}{(3 k) !}=\frac{1}{k ! 3^{k}}$, which yields [5, Theorem $3.1]$.

\section{Concluding remarks}

In this paper, by introducing the CLS -sequence, we present a combinatorial expansion of LS $(n+k, n)$. It should be noted that the CLS-sequence has several other variants.

For an alphabet $A$, let $\mathbb{Q}[[A]]$ be the rational commutative ring of formal Laurent series in monomials formed from letters in $A$. Following Chen [4], a context-free grammar over $A$ is a function $G: A \rightarrow \mathbb{Q}[[A]]$ that replace a letter in $A$ by a formal function over $A$. The formal derivative $D=D_{G}: \mathbb{Q}[[A]] \rightarrow \mathbb{Q}[[A]]$ is defined as follows: for $x \in A$, we have $D(x)=G(x)$; for a monomial $u$ in $\mathbb{Q}[[A]], D(u)$ is defined so that $D$ is a derivation, and for a general element $q \in \mathbb{Q}[[A]], D(q)$ is defined by linearity. The reader is referred to [10] for recent results on context-free grammars.

As a variant of the CLS-sequence, we now introduce a marked scheme for LegendreStirling set partitions. Given a set partition $\sigma=B_{1} B_{2} \cdots B_{k} B_{0} \in \mathcal{L} \mathcal{S}(n, k)$, where $B_{0}$ is the zero box of $\sigma$. We mark the box vector $\left(B_{1}, B_{2}, \ldots, B_{k}\right)$ by the label $a_{k}$. We mark 
any box pair $\left(B_{i}, B_{j}\right)$ by a label $b$ and mark any box pair $\left(B_{s}, B_{0}\right)$ by a label $c$, where $1 \leqslant i<j \leqslant k$ and $1 \leqslant s \leqslant k$. Let $\sigma^{\prime}$ denote the Legendre-Stirling set partition that generated from $\sigma$ by inserting $n+1$ and $\overline{n+1}$. If $n+1$ and $\overline{n+1}$ are in the same box, then $\sigma^{\prime}=B_{1} B_{2} \cdots B_{k} B_{k+1} B_{0}$, where $B_{k+1}=\{n+1, \overline{n+1}\}$. This case corresponds to the operator $a_{k} \rightarrow a_{k+1} b^{k} c$.

If $n+1$ and $\overline{n+1}$ are in different boxes, then we distinguish two cases:

(i) Given a box pair $\left(B_{i}, B_{j}\right)$, where $1 \leqslant i<j \leqslant k$. We can put $n+1$ (resp. $\overline{n+1}$ ) into the box $B_{i}$ and put $\overline{n+1}$ (resp. $n+1$ ) into the box $B_{j}$. This case corresponds to the operator $b \rightarrow 2 b$.

(ii) Given a box pair $\left(B_{i}, B_{0}\right)$, where $1 \leqslant i \leqslant k$. We can put $n+1$ (resp. $\overline{n+1}$ ) into the box $B_{i}$ and put $\overline{n+1}$ (resp. $n+1$ ) into the zero box $B_{0}$. Moreover, we mark each barred entry in the zero box $B_{0}$ by a label $z$. This case corresponds to the operator $c \rightarrow(1+z) c$.

Let $A=\left\{a_{0}, a_{1}, a_{2}, a_{3}, \ldots, b, c\right\}$ be a set of alphabet. Following the above marked scheme, we consider the grammars

$$
G_{k}=\left\{a_{0} \rightarrow a_{1} c, a_{1} \rightarrow a_{2} b c, \ldots, a_{k-1} \rightarrow a_{k} b^{k-1} c, b \rightarrow 2 b, c \rightarrow(1+z) c\right\},
$$

where $k \geqslant 1$. It is a routine check to verify that

$$
D_{n} D_{n-1} \cdots D_{1}\left(a_{0}\right)=\sum_{k=1}^{n} \operatorname{JS}_{n}^{k}(z) a_{k} b^{\left(\begin{array}{c}
k \\
2
\end{array}\right)} c^{k}
$$

Therefore, it is clear that for $n \geqslant k$, the number $\operatorname{JS}_{n}^{k}(z)$ is a polynomial of degree $n-k$ in $z$, and the coefficient $z^{i}$ of $\operatorname{JS}_{n}^{k}(z)$ is the number of Legendre-Stirling partitions in $\mathcal{L} \mathcal{S}(n, k)$ with $i$ barred entries in zero box, which gives a grammatical proof of [8, Theorem 2].

We end our paper by proposing the following.

Conjecture 9. For any $k \geqslant 1$, the polynomial $\gamma_{k}(x)$ has only real zeros. Set

$$
\gamma_{k}(x)=\gamma(k, 3 k) x^{k+2} \prod_{i=1}^{2 k-2}\left(x-r_{i}\right), \gamma_{k+1}(x)=\gamma(k+1,3 k+3) x^{k+3} \prod_{i=1}^{2 k}\left(x-s_{i}\right),
$$

where $r_{2 k-2}<r_{2 k-3}<\cdots<r_{2}<r_{1}$ and $s_{2 k}<s_{2 k-1}<s_{2 k-2}<\cdots<s_{2}<s_{1}$. Then

$$
s_{2 k}<r_{2 k-2}<s_{2 k-1}<r_{2 k-3}<s_{2 k-2}<\cdots<r_{k}<s_{k+1}<s_{k}<r_{k-1}<\cdots<s_{2}<r_{1}<s_{1},
$$

in which the zeros $s_{k+1}$ and $s_{k}$ of $\gamma_{k+1}(x)$ are continuous appearance, and the other zeros of $\gamma_{k+1}(x)$ separate the zeros of $\gamma_{k}(x)$.

\section{Acknowledgements}

The authors thank the referee for his valuable suggestions. 


\section{References}

[1] G.E. Andrews, E.S. Egge, W. Gawronski, L.L. Littlejohn, The Jacobi-Stirling numbers, J. Combin. Theory Ser. A, 120(1):288-303, 2013.

[2] G.E. Andrews, W. Gawronski, L.L. Littlejohn, The Legendre-Stirling numbers, Discrete Math., 311:1255-1272, 2011.

[3] G.E. Andrews, L.L. Littlejohn, A combinatorial interpretation of the LegendreStirling numbers, Proc. Amer. Math. Soc., 137:2581-2590, 2009.

[4] W.Y.C. Chen, Context-free grammars, differential operators and formal power series, Theoret. Comput. Sci., 117:113-129, 1993.

[5] E.S. Egge, Legendre-Stirling permutations, European J. Combin., 31(7):1735-1750, 2010.

[6] W.N. Everitt, L.L. Littlejohn, R. Wellman, Legendre polynomials, Legendre-Stirling numbers, and the left-definite analysis of the Legendre differential expression, $J$. Comput. Appl. Math., 148(1):213-238, 2002.

[7] W.N. Everitt, K.H. Kwon, L.L. Littlejohn, R. Wellman, G.J. Yoon, Jacobi-Stirling numbers, Jacobi polynomials, and the left-definite analysis of the classical Jacobi differential expression, J. Comput. Appl. Math., 208:29-56, 2007.

[8] Y. Gelineau, J. Zeng, Combinatorial interpretations of the Jacobi-Stirling numbers, Electron. J. Combin., 17:\#R70, 2010.

[9] I.M. Gessel, Z. Lin, J. Zeng, Jacobi-Stirling polynomials and P-partitions, European J. Combin., 33:1987-2000, 2012.

[10] S.-M. Ma, J. Ma, Y.-N. Yeh, B.-X. Zhu, Context-free grammars for several polynomials associated with Eulerian polynomials, Electron. J. Combin., 25(1):\#P1.31, 2018.

[11] M. Merca, A connection between Jacobi-Stirling numbers and Bernoulli polynomials, J. Number Theory., 151:223-229, 2015.

[12] P. Mongelli, Combinatorial interpretations of particular evaluations of complete and elementary symmetric functions, Electron. J. Combin., 19(1):\#P60, 2012. 